\title{
Research Paper \\ Relationship Between Self-Regulation and Balance-Confidence in Active and Inactive Elderly Men
}

\author{
*Fatemeh Sadat Hosseini ${ }^{1}$, Omid Hatamnezhad ${ }^{1}$ \\ 1. Department of Motor Behavior and Sport Management, Faculty of Sport Sciences, Urmia University, Urmia, Iran.
}

Crtation: Hosseini FS, Hatamnezhad O. [Relationship Between Self-Regulation and Balance-Confidence in Active and Inactive Elderly Men (Persian)]. Iranian Journal of Ageing. 2018; 12(4):470-481. https://doi.org/10.21859/sija.12.4.470

https://doi.org/10.21859/sija.12.4.470

Received: 07 Aug. 2017 Accepted: 27 Oct. 2017

Key words:

Older adults, Balance confidence, Positive performance, Self-regulation

\begin{abstract}
Objectives The aim of this study was to examine the relationship between self-regulation and balance confidence among the active and in-active elderly in the city of Mahabad.

Methods \& Materials The cross-correlational research included elderly people aged 60 to 85 years and living in Mahabad city. A total of 93 active elderly and 93 in-active elderly were randomly selected as sample. The Balance Confidence questionnaire and Self-Regulation questionnaire were used to gather data. The Kolmogorov-Smirnov test, Pearson correlation, multiple regressions, and independent $t$ test were used to analyze the collected data. This study was performed using SPSS software.

Results The results showed that balance confidence had a positive and significant relationship with all the dimensions of self-regulation in the elderly $(P \leq 0.05)$. Other than the disclosure feelings component $(P=0.250)$, other dimensions of self-regulation can effect balance confidence. It was also found that there is a significant difference between the active (balance confidence: $3.2446 \pm 0.63735$; self-regulation: $3.8017 \pm 0.75943$ ) and in-active (balance confidence: $2.3468 \pm 0.69454$; self-regulation: $2.8208 \pm 0.79782$ ) elderly ( $\mathrm{P} \leq 0.05)$.

Conclusion This study confirms that lack of self-regulation in the elderly can lead to decreased physical activity and restrictions. It is suggested that self-regulation should be emphasized in the elderly so that they can overcome their environmental issues and enhance their balance confidence.
\end{abstract}

\section{Extended Abstract}

\section{Objective}

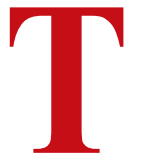

he main consequences of balance problems in the elderly are falling and the fear of falling; these two consequences affect everyday life activities and the ability to move in home and community $[1,2]$. The fear of falling also leads to depression and lower quality of life in the elderly [3]. Studies on balance confidence have reported its relationship with some functional abilities and personal characteristics in the elderly $[4,5]$. In addition to the relationship between balance confidence and balance performance $[6$, 7], the relationship between balance confidence and physical activity [8] has also been confirmed. One of the factors that can contribute to balance confidence in the elderly is self-regulation. Self-regulation refers to the processes based on which people control their thoughts, feelings, and actions [9]. Therefore, the present study aims to investigate the relationship between

* Corresponding Author:

Fatemeh Sadat Hosseini, PhD

Address: Department of Motor Behavior and Sport Management, Faculty of Sport Sciences, Urmia University, Urmia, Iran.

Tel: +98 (912) 9469087

E-mail: fhosseini2002@yahoo.com 
self-regulation and balance confidence in active and inactive older adults in Mahabad city.

\section{Methods \& Materials}

The present study was an applied research in terms of objective and also a descriptive-correlational one in terms of data collection. The entire research process was conducted under the supervision of the local ethics committee of the Department of Sports Sciences in Uremia University. The study population consisted of all older adults aged 60-85 years old in Mahabad city. A sample of 93 active and 93 inactive elders was selected using available sampling method. Here, inactive elderly subjects referred to those elderly individuals who did not have any regular individual or group physical activity in the last few years. The active elderly people were those elderly individuals who performed at least one physical activity for at least one hour per day for three days a week in the last two consecutive years.

A demographic questionnaire, balance confidence scale, and self-regulation questionnaire were used to collect the data needed for this study and measure the research variables. Activities-specific Balance Confidence Scale by Powell and Myers [10] has 16 questions in which the respondents are asked to assess their confidence when performing a range of daily life activities. This scale has been evaluated in Iran by Khajavi [11], and its structural validity has been reported using exploratory and confirmatory factor analysis. The retest reliability, Cronbach's alpha, and correlation coefficient were $0.82,0.98$, and 0.98, respectively. Also, the Self-Regulation Inventory (SRI-25) is a 25-point test that measures self-regulation in five areas of Positive Actions, Controllability, Expression of Feelings and Needs, Assertiveness, and Well-Being Seeking on a 5-point Likert scale. Data analysis was performed using Kolmogorov-Smirnov Test, single sample t-test, independent t-test, Pearson correlation coefficient, multiple regression analysis. All statistical operations were performed using SPSS version 22.

\section{Results}

Active elderly showed better performance in balance confidence and self-regulation compared with the inactive elderly. This may be because single-sample ttest results for self-regulation $(\mathrm{P}=0.053)$ and balance confidence $(\mathrm{P}=0.056)$ were not significant among inactive people while it showed a significant difference for self-regulation ( $\mathrm{P}=0.001)$ and balance confidence $(\mathrm{P}=0.001)$ in the active elderly.

The results showed that there is a positive and significant correlation between self-regulation and balance confidence among active and inactive elderly people, and this coefficient was about 0.84 . In the various dimensions of self-regulation, this relationship was positive and significant. There was a positive and significant correlation between balance confidence and positive actions (0.75), controllability (0.64), expression of emotions and needs $(0.55)$, assertiveness $(0.75)$, and wellbeing seeking (0.62) among active and inactive elderly people. Overall, the results showed that all dimensions of self-regulation have a positive and significant relationship with balance confidence in the elderly.

Except for the variable of expression of emotions and needs, other dimensions of self-regulation can affect the balance confidence. The analysis of multiple linear regression in a simultaneous manner suggests that among the five predictors of balance confidence, all dimensions of self-regulation, other than the expression of emotions, have significant weights in the regression predictor equation. Using non-standard regression coefficients and the scores of positive actions, well-being seeking, assertiveness and controllability, the regression equation to predict balance confidence can be written as follows.

Balance Confidence $=0.12+0.30$ (positive actions) +0.15 (well-being seeking) +0.14 (assertiveness) +0.21 (controllability)

0.12 in the equation is a constant or intercept. Regarding the beta coefficient obtained, it can be stated that the variable of positive actions has the most effect on balance confidence of the active and inactive elderly people.

\section{Conclusion}

It is suggested that the elderly themselves can overcome environmental problems. Self-regulation structures provide the most evidence for physical activity modification, and they tend to adhere to exercise by increasing the pleasure derived from exercise [12]. This adherence to exercise and physical activity can increase balance confidence and reduce the level of fear of falling and also prevent the lack of physical inactivity $[7,8,13]$. It seems that fear of falling in older people, which leads to lower self-esteem and self-regulation, can be considered as a barrier to proper physical activity [14]. However, proper physical activity in the 
aging period is one of the main factors of successful aging [15]. As a result, it is better to focus on barriers to create an active elderly lifestyle, such as fear of falling, when designing interventions.

\section{Acknowledgments}

Faculty of Sport Sciences, Urmia University has financially supported the present research.

\section{Conflict of Interest}

The authors declared no conflicts of interest. 


\title{
رابطه بين خودتنظيمى با اعتماد به تعادل در سالمندان مرد فعال و غير فعال
}

\author{
"فاطمه سادات حسينى'، اميد حاتمثزراد' \\ ا- كروه رفتار حركتى و مديريت ورزشى، دانشكده علوم ورزشى، دانشُكاه اروميه، اروميه، ايران.
}

\begin{abstract}
حكبد
الهدالف هدف يروهش حاضر بروسى ارتباط بين خودتنظيمى با اعتماد به تعادل در سالمندان فعال وغير فعال ششهرستان مهاباد بود.

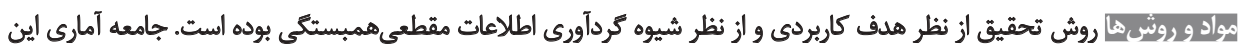

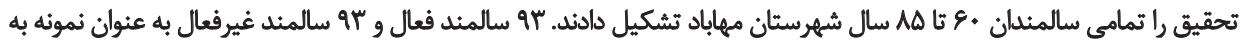

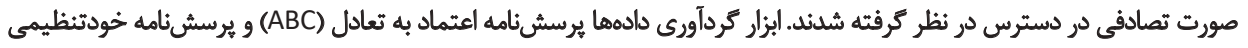

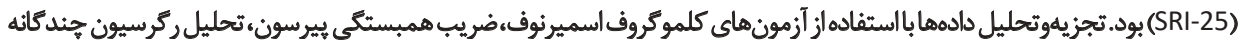

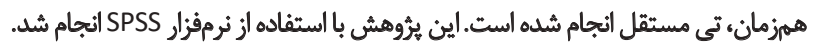

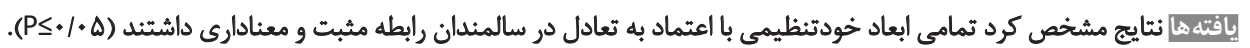

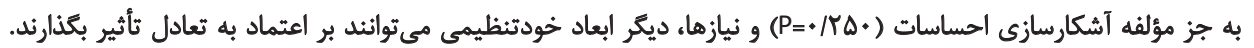

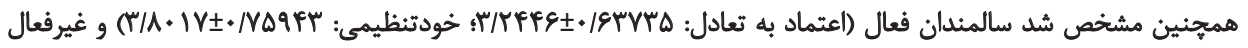

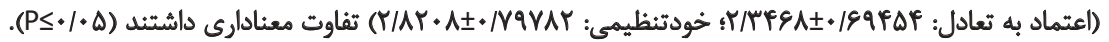

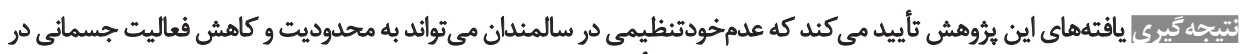

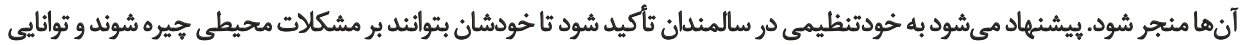
اعتماد به تعادل در آنها ارتقا يابلد.
\end{abstract}

تاريخ دريافت: 19 مرداد وهوباد

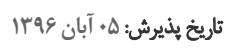

dotio

شد [9]. علاوه بر اين، تحقيقات مرورى بيانكر اين مطلب هستيند

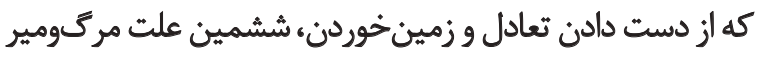

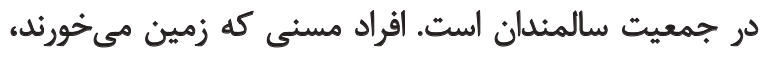

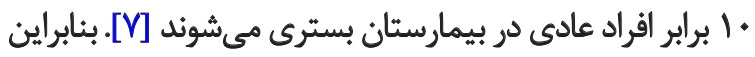

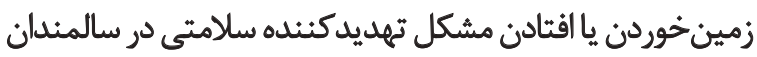

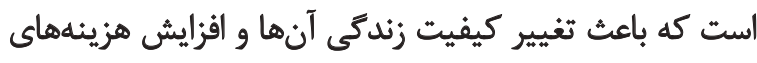

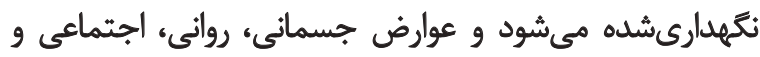

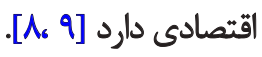

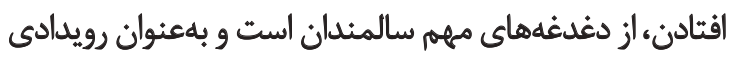

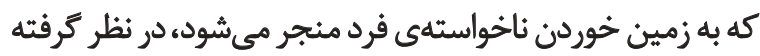

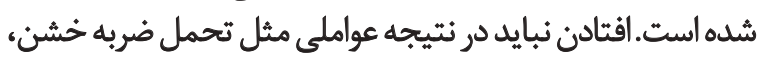

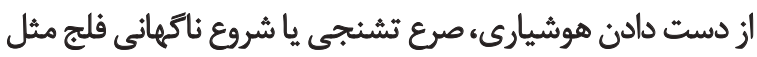

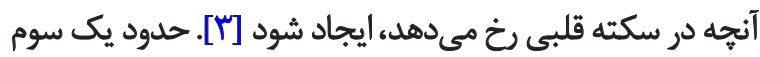

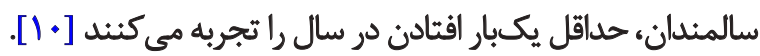

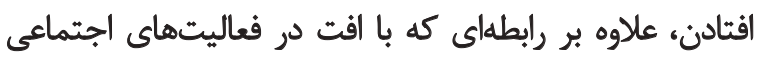

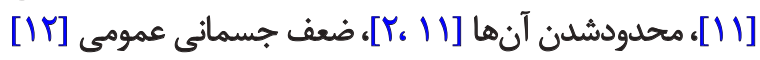

افزايش اميد به زندكى و كاهش ميزان بارورى باعث افزايش

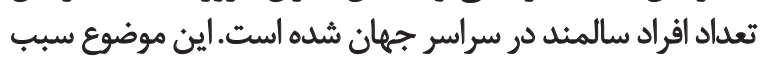

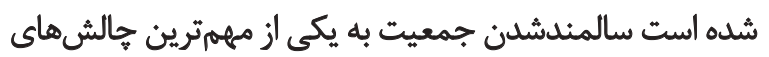

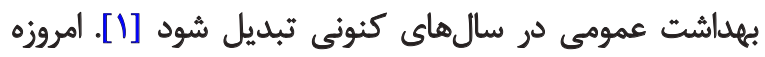

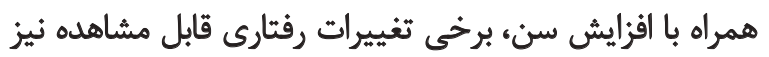

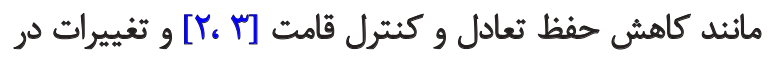

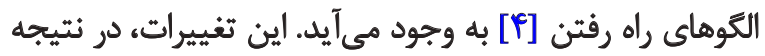

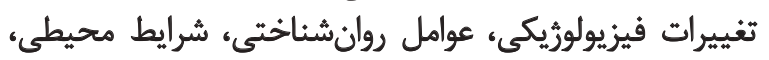

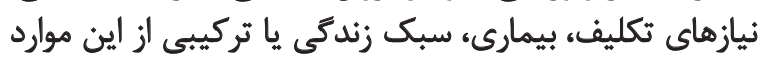
بروز مي كند [ه].

يكى از عواقب برجسته مشكلات تعادلى، افتادن و ترس از از

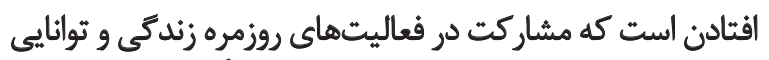

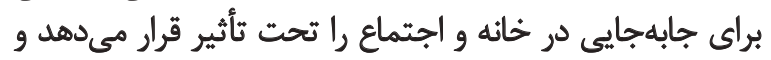

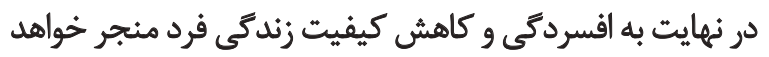


مي شود. بعد جهارم قاطعيت است كه شامل دفاع از منافع خود،

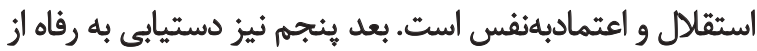

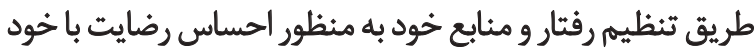

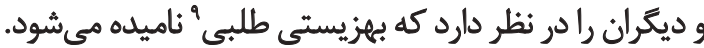
خواجوى و همكاران [بr] نشان دادند كه بين تغييرات در

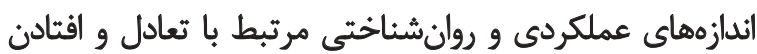

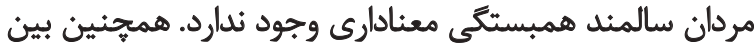

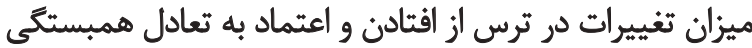

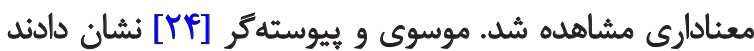

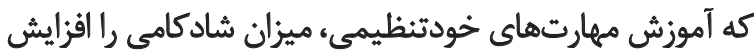

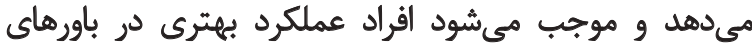

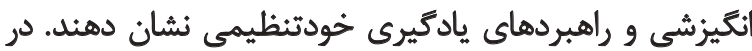

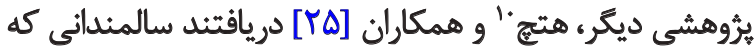

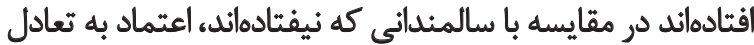

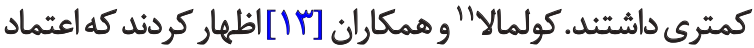

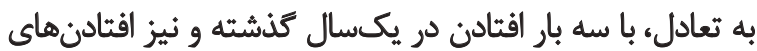

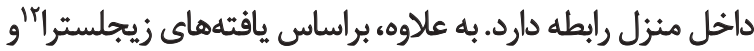

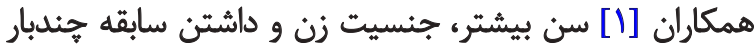

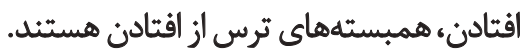

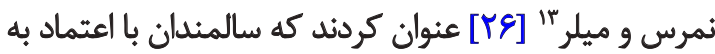

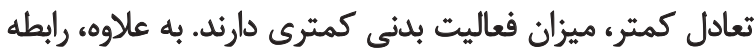

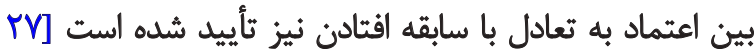

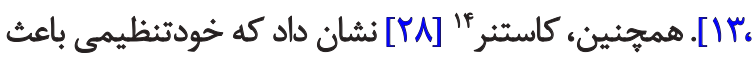

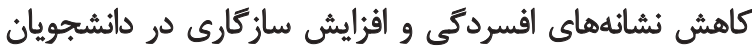

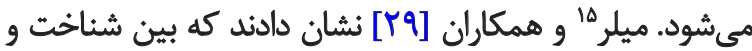

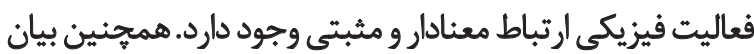

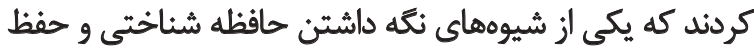

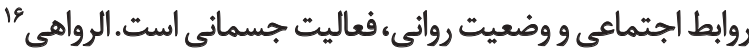

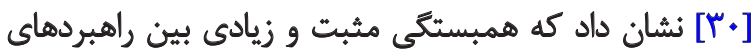

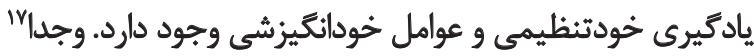

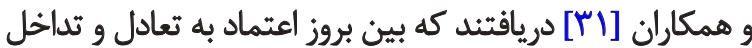

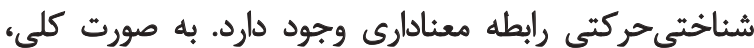

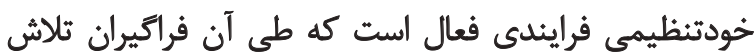

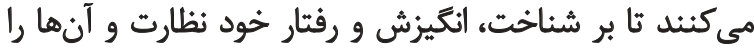

8. Decisiveness

9. Seeking Welfare

10. Hatch

11. Kulmala

12. Zijlstra

13. Nemmers and Miller

14. Koestner

15. Miller

16. Al-rawahi

17. Wajda
و كاهش كيفيت زندكى [Y] دارد، موجب كاهش اعتماد به تعادل'

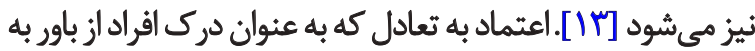

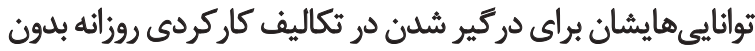

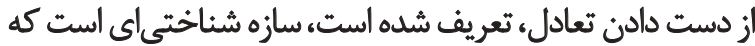

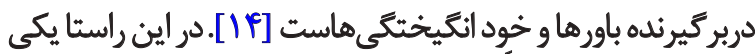

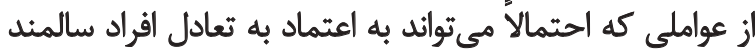

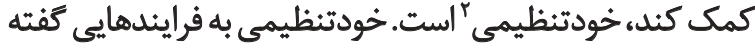

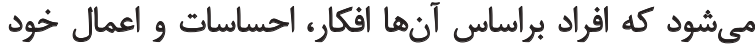

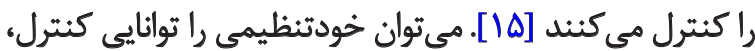

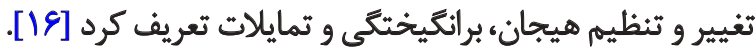

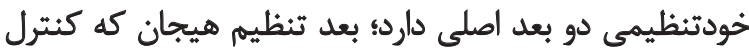

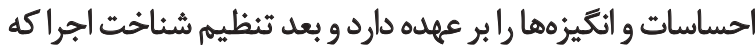

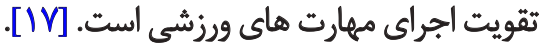

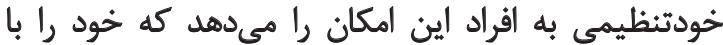

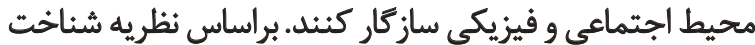

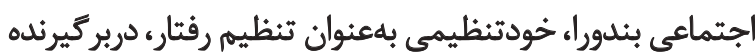

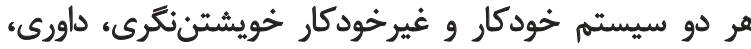

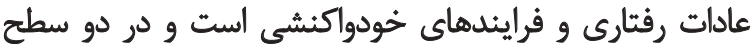

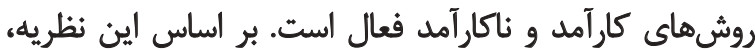

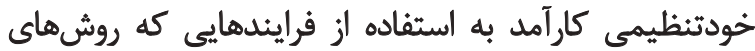

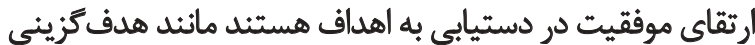

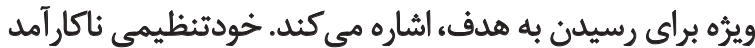

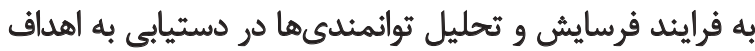

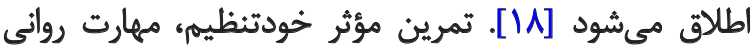

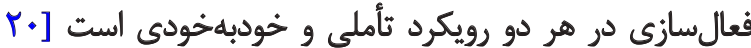

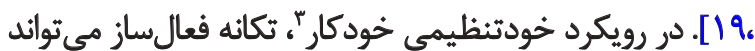

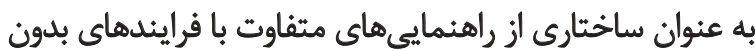

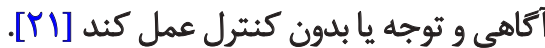

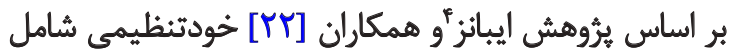

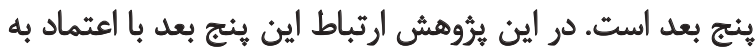

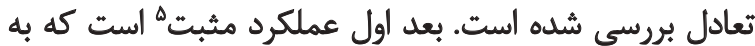

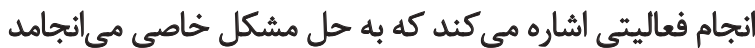

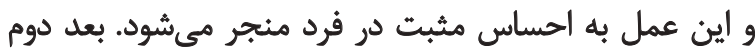

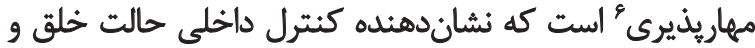

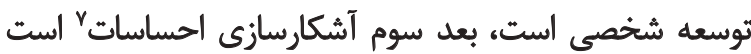

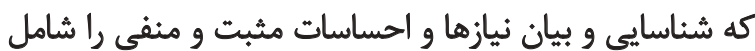

1. Balance Confidence

2. Self-regulation

3. Automatic self-regulation

4. Ibánez

5. Positive performance

6. Inhibition

7. Disclosure feelings 


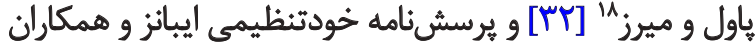

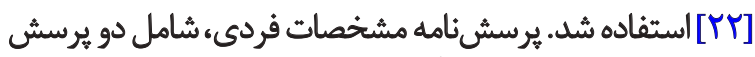

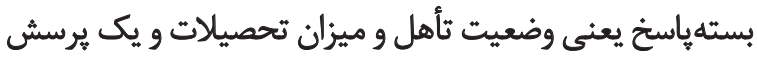

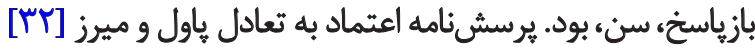

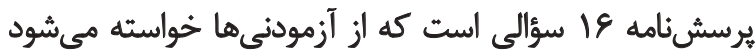

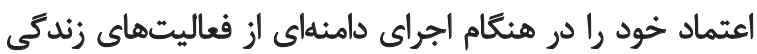

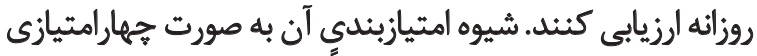

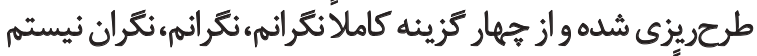
و كاملأنكرانم تشكيل شده است.

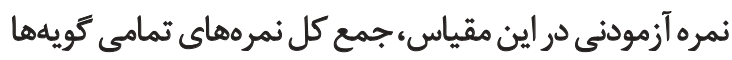

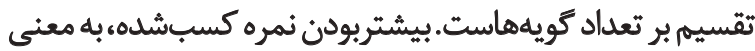

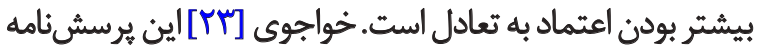

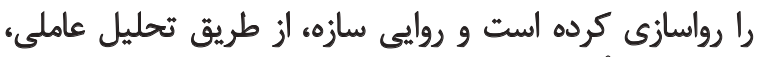

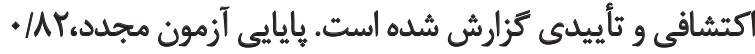

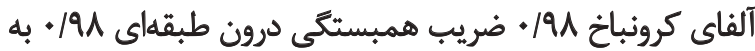

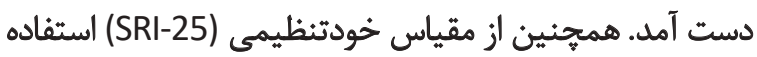

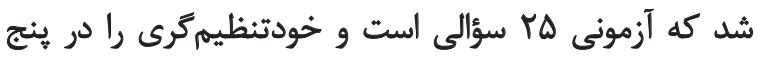

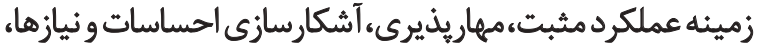

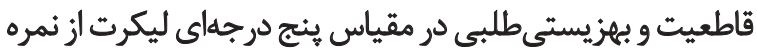

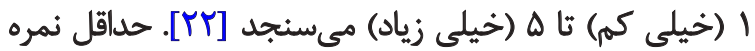

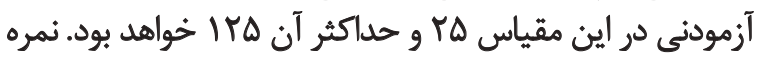

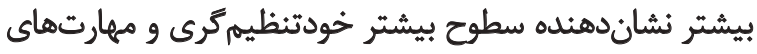

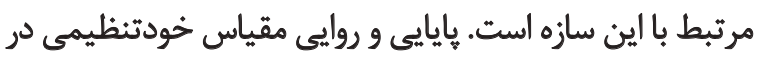

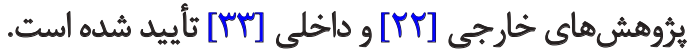

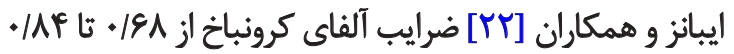

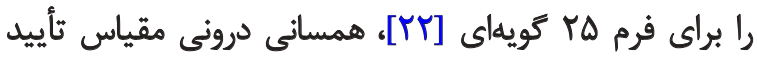

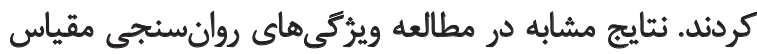

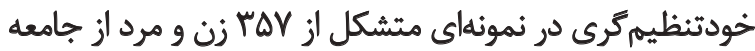

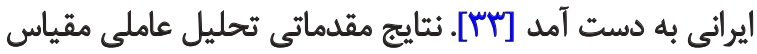

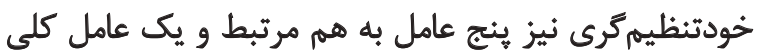

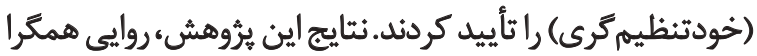

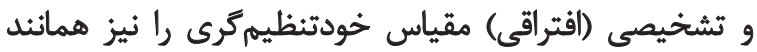
مطالعات مشابه خارجى [افتراقى مقيأي خوديد كرد.

براى طبقلهبندى نمرات خام، طراحى جدول توزيع فراوانى و

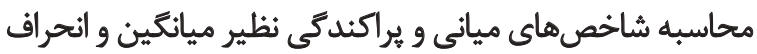

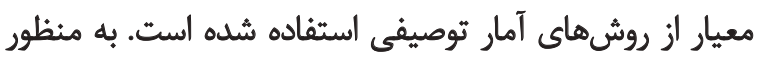

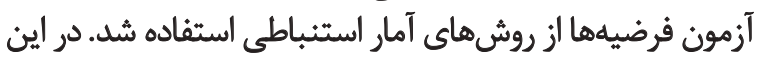

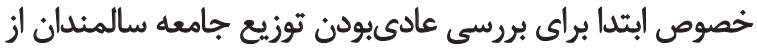

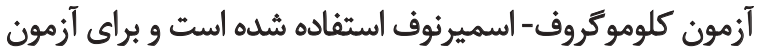

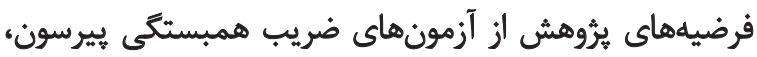

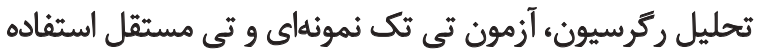

تنظيم و كنترل كنند. سازههاى خودتنظيمى ورزشى بيشترين

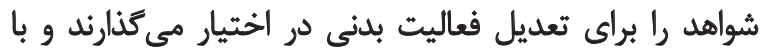

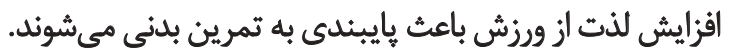
يثروهش حاضر معطوف به حوزه مهمى است كه ممكن است باعث

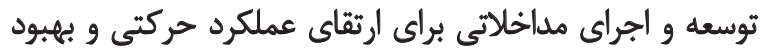

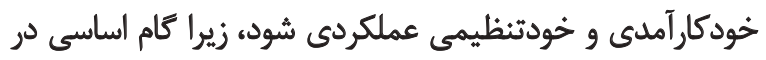

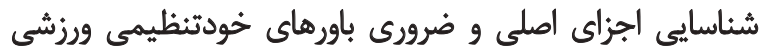

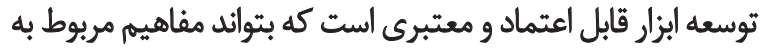

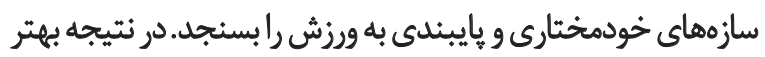

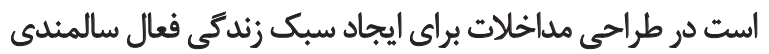

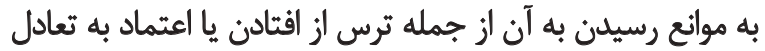

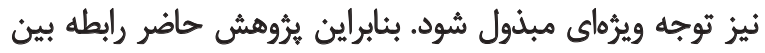

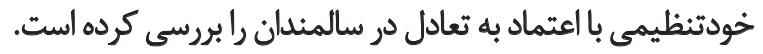

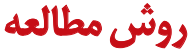

يروهش حاضر از نظر هدف "(كاربردى)، و از نظر شيوه

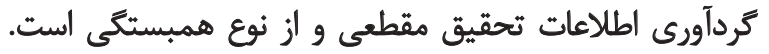

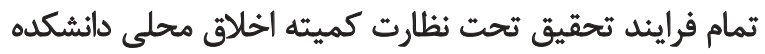

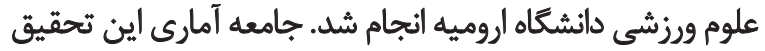

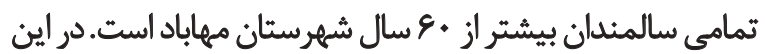

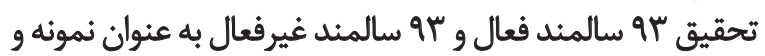

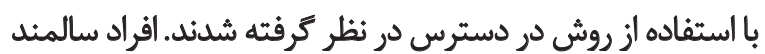

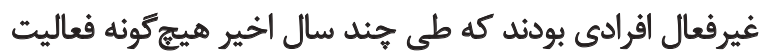

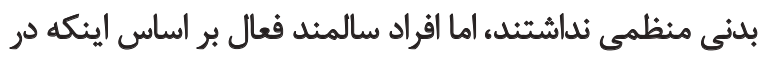

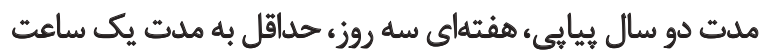

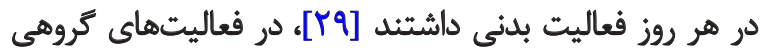

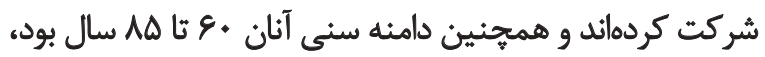

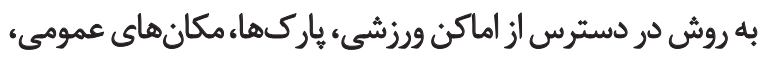

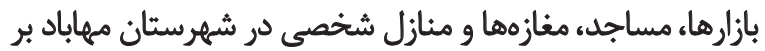

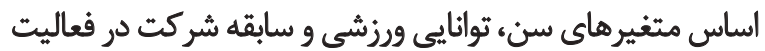
بدنى در مطالعه حاضر انتخاب شدند

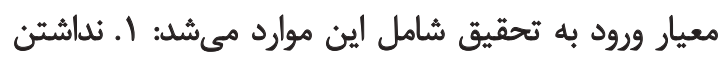

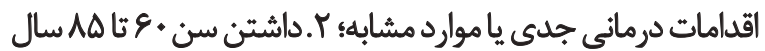

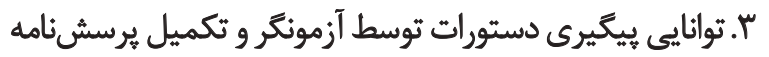

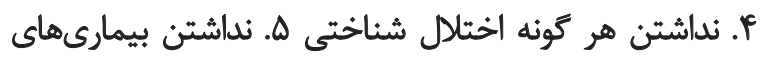

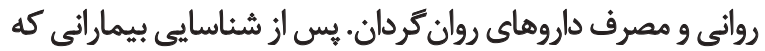

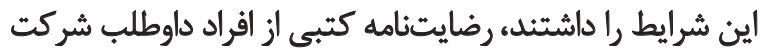

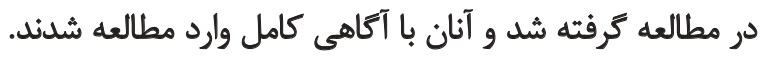

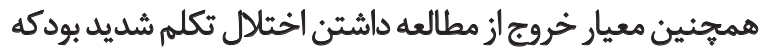

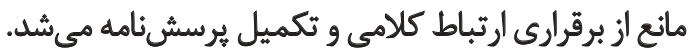

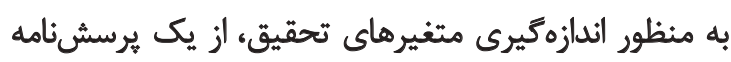

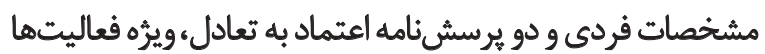




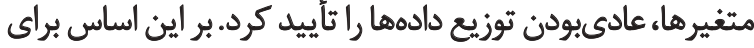

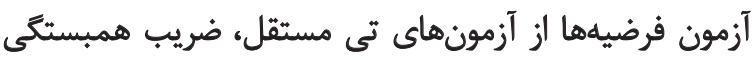

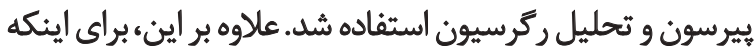

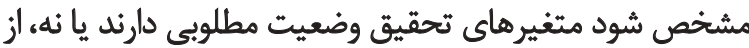

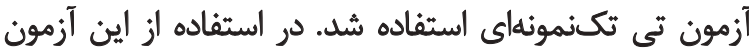

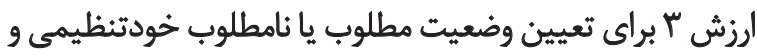
اعتماد به تعادل در نظر ترفته شده است.

يافتههاى تي تكنمونهاي مشخص كرد سالمندان فعال در

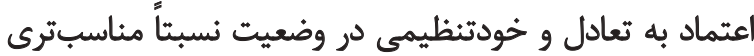

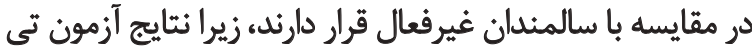

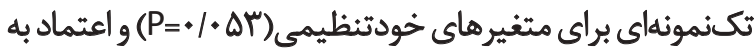

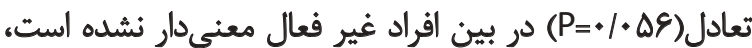

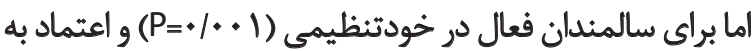

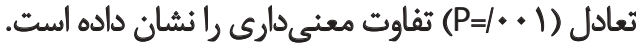

براى بررسى ارتباط خودتنظيمى و ابعاد آن با اعتماد به تعادل در

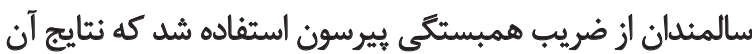

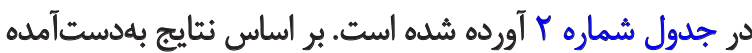

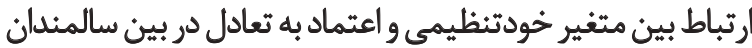

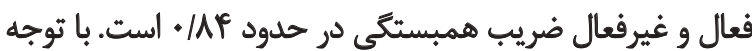

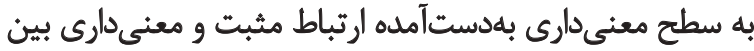

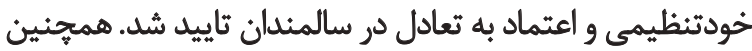

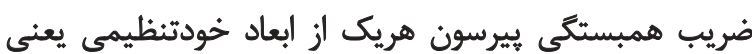

شده است. تمامى عمليات با نرمافزار SPSS نسخه كب انجام شد.

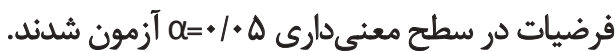

يافتهها

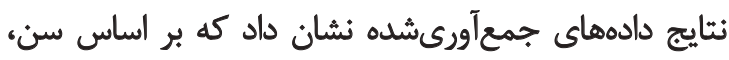

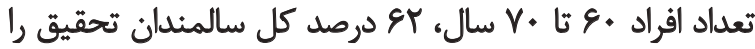

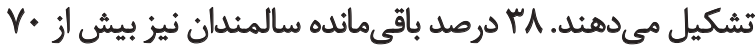

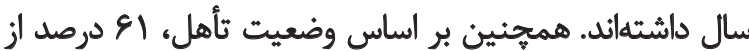

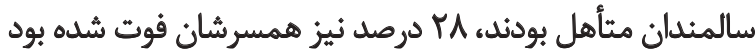

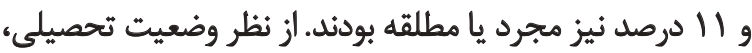

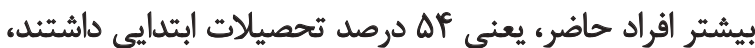

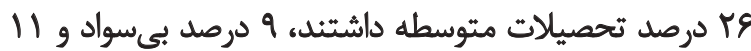

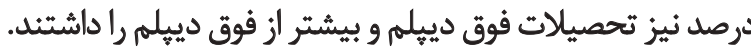

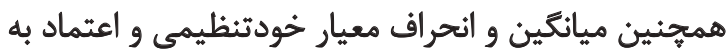

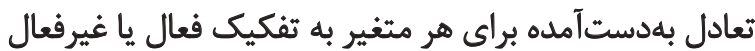

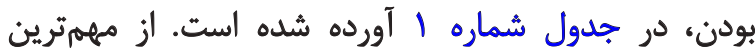

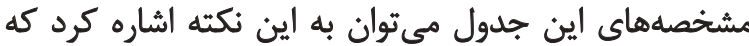

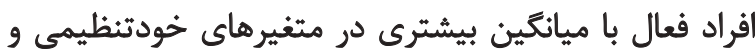
اعتماد به تعادل هستيند.

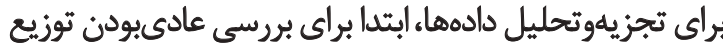

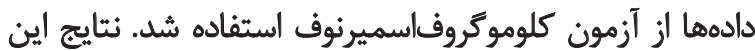

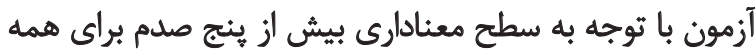

جدول ا. ميانكَين و انحراف معيار بررسى وضعيت خودتثظيمى و اعتماد به تعادل سالمندان فعال و غيرفعال

\begin{tabular}{|c|c|c|}
\hline غيرفعال & فعال & متغيرها \\
\hline T/MFEA +/EqFAF & r/MTEE = / RTM" & اعتماد به تعادل \\
\hline r/MITI.MVVE & $r / N E \cdot A \pm \cdot M T V I r$ & عملكرد مثبت \\
\hline 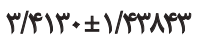 & 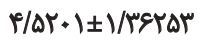 & آثكارسازى \\
\hline T/FIFA & 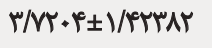 & بهزيستى \\
\hline 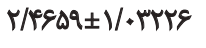 & $r / \Delta r+\Delta \pm / / 1 F V$. & قاطلعيت \\
\hline r/erVat.NaEri & $r / F V E V \pm$. $F A E Y \mid$ & مهاريذيرى \\
\hline 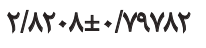 & $r / A \cdot I V \pm=/ N \Delta Q P T$ & خودتنظيمى \\
\hline
\end{tabular}

il

جدول r. ضريب همبستكى ييرسون بين اعتماد به تعادل و خودتنظيمى

\begin{tabular}{|c|c|c|c|c|c|c|}
\hline بهزيستى طلبى & قاطعيت & آشكارسازى & مهاريذيرى & عملكردمثبت & خودتثظيمى & \\
\hline - $18 \pi T^{2}$ & - Nare: & $\cdot(\Delta Q)^{* * *}$ & $.194 . *$ & - Mpq ** & $\cdot / A f * *$ & \\
\hline.$/ .+1$ & $+1+.1$ & $+1+\infty 1$ & $. \%+1$ & $.10+1$ & $+1+* 1$ & اعتماد به تعادل \\
\hline$W$ & $W^{E}$ & $w$ & W & We & WE & \\
\hline
\end{tabular}


جدول r.نتايج تحليل ركرسيون جيندكائه همزمان

\begin{tabular}{|c|c|c|c|c|c|c|}
\hline \multirow{2}{*}{ معنى دارى } & \multirow{2}{*}{ مقدار T } & \multirow{2}{*}{ ضريب اسثاندارد } & \multicolumn{2}{|c|}{ ضريب غيراستاندارد } & \multirow{2}{*}{ مدل } & \\
\hline & & & خطاى معيار & بثا & & \\
\hline . &.$/ 9 \cdot 1$ & & . & H Ir. & (ثابت) & \\
\hline $.1 . .1$ & $\Delta / T q 1$ &.$/ M \mathrm{M}$ & $\cdot 1 \cdot \Delta V$ & $\cdot \pi+1$ & عملكرد هثبت & \\
\hline.$/ T \Delta$ & $1 / 100$ & $\cdot 1 \cdot \Delta f$ & $.1 \cdot 50$ & $.1 . r q$ & آشكارسازى & \\
\hline$+1+.1$ & P/AYA & . RAF & 1.41 &.$/ 101$ & بهزيستى & 1 \\
\hline 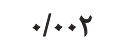 & $m / 1.9$ & $\cdot M M$ & $1 . P \Delta$ & /lit. & قاطعيت & \\
\hline $.1 .+1$ & r/VEV & ./MTE & $.1 . \Delta 8$ & - Mir & ههاريذيرى & \\
\hline
\end{tabular}

جدول †. مقايسه خودتنظيمى و اعتماد به تعادل سالمندان فعال و غيرفعال

\begin{tabular}{|c|c|c|c|}
\hline معنى دارى & $\mathrm{t}$ & درجه آزادى & سالمندان فعال و غيرفعال \\
\hline $.1 . .1$ & NAM & Mf & خودود تئظيميى \\
\hline $.1 . .1$ & VINA & MF & اعتماد به تعادل \\
\hline
\end{tabular}

乩

عدد ب / / در فرمول عدد ثابت يا همان عرض از مبداً است.

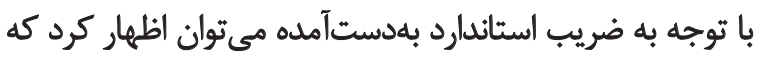

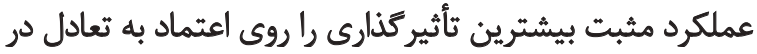

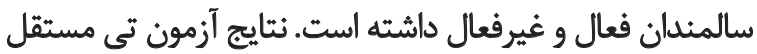

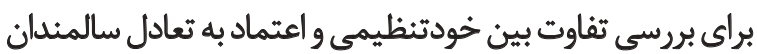

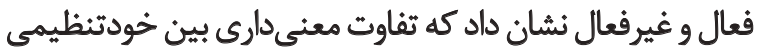

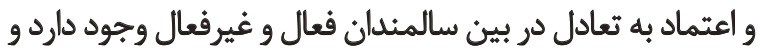

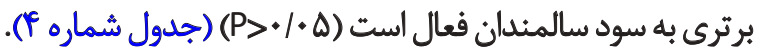

\section{بحث}

هدف بروهش حاضر تبيين ارتباط بين ابعاد خودتنظيمى با

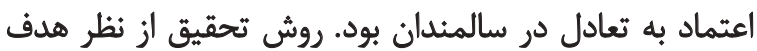

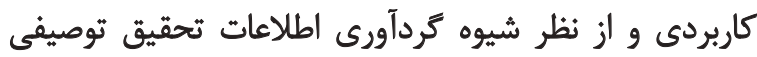

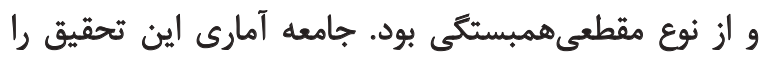

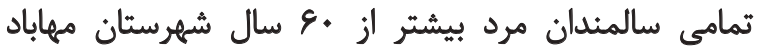

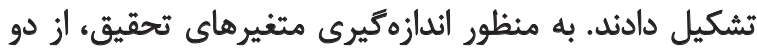

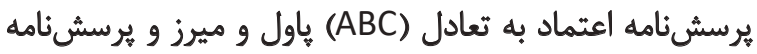

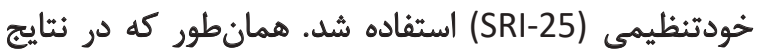

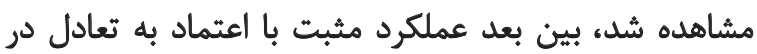

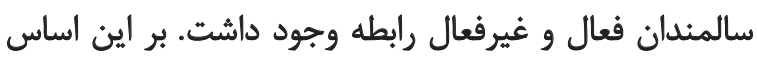

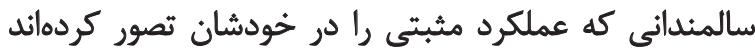

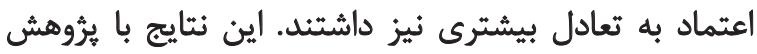

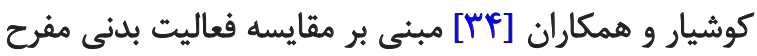
و معمولى بر تعادل و ترس از سقوط سالمندان همسو است.

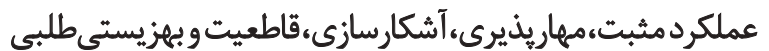

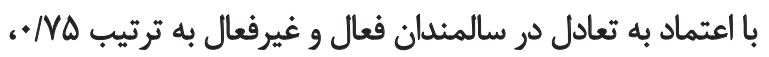

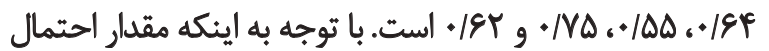

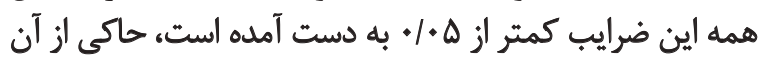

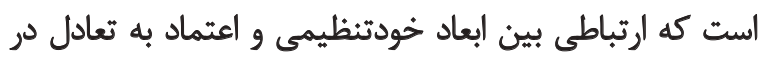
سالمندان فعال و غيرفعال وجود دارد.

براي بررسى اينكه ابعاد خودتنظيمى مي توانند بر تغييرات

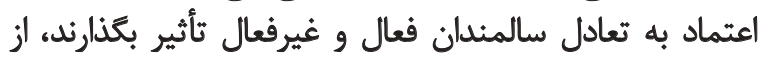

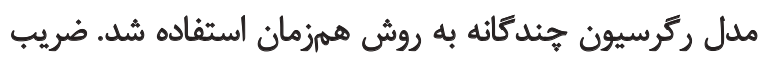

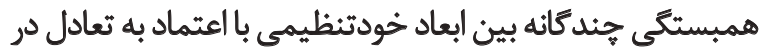

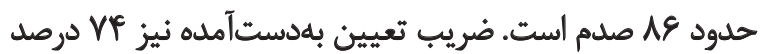

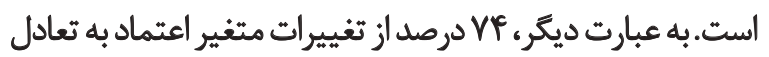
ازز ابعاد خودتنظيمى ناشى شده است.

نثايج بهدستآمده از تحليل ركرسيون خطى خيندكانه به روش

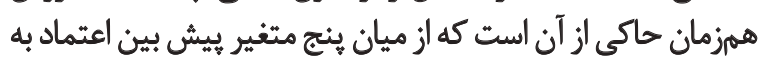

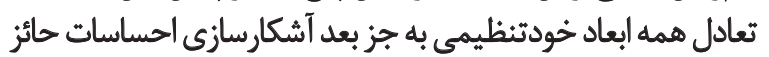

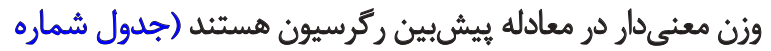

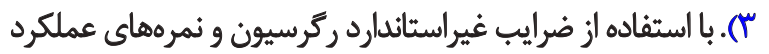

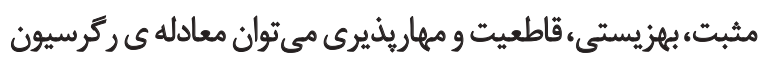

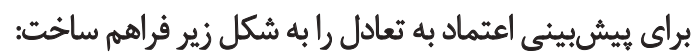

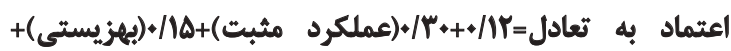

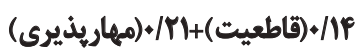


شادكامى ايشان مىشود. همجينين مي توانند بر احساسات و ورافي

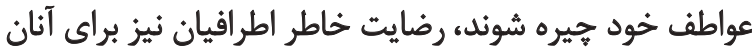

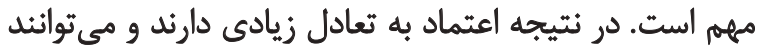
بر ترس از افتادن غلبه كنيند.

همجنين نتايج بهدستآمده از تحليل ركرسيون خطى

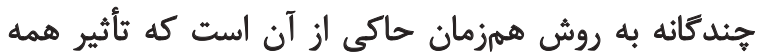

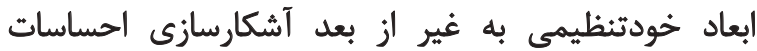

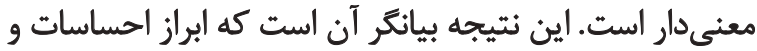

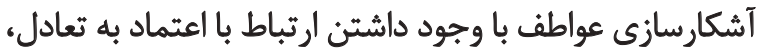

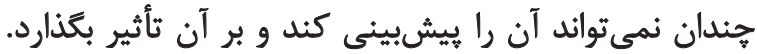

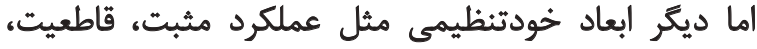

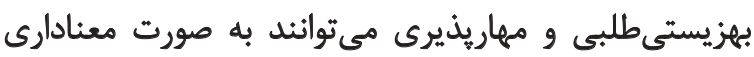

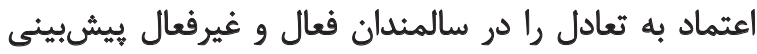

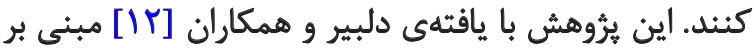

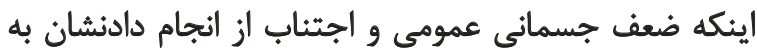

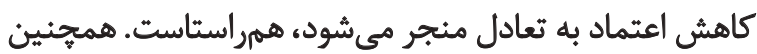
با نتايج يافتهاي بويد و استيون [Y]، كولمالاو همكاران [IT) همسو است. علاوه بر اين، نتايج آزمون تى مستقل نشان داد كه بين

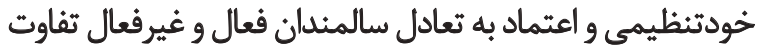

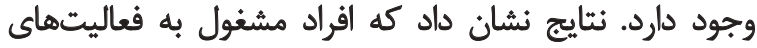

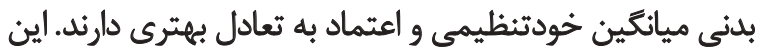

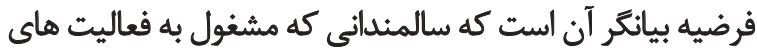

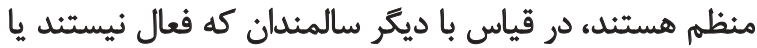

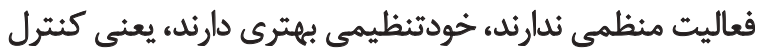

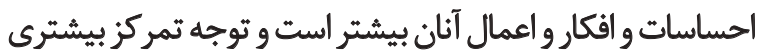

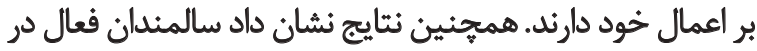

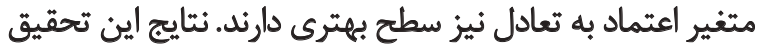

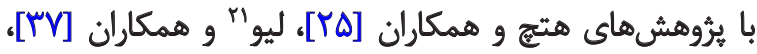

$$
\text { نمرس و ميلر [عب] همراستاست. }
$$

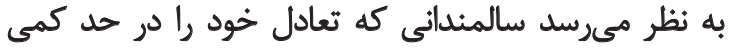

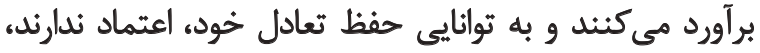

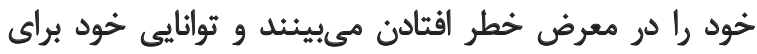

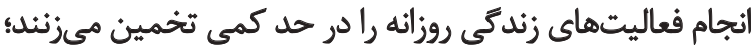

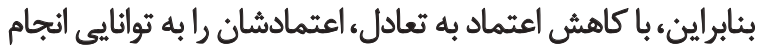

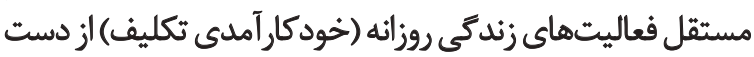

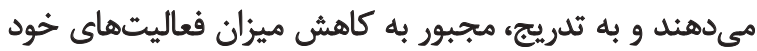

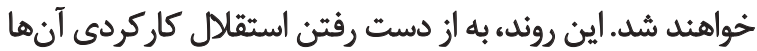

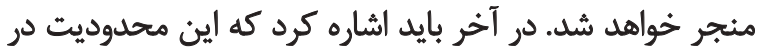

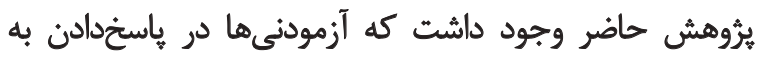

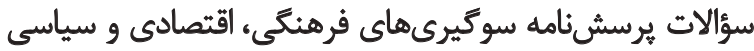

از طرف ديكَ، بين بعد مهاريذيرى با اعتماد به تعادل در

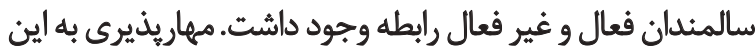

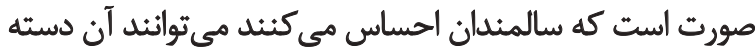

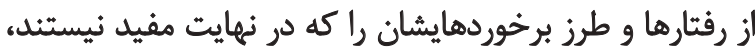

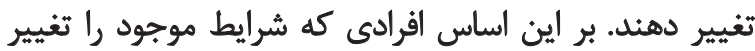

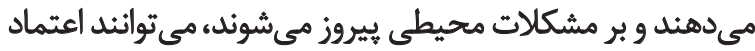

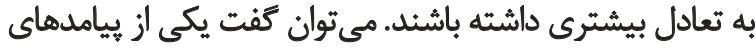

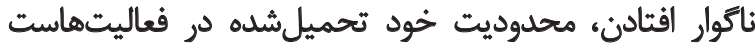

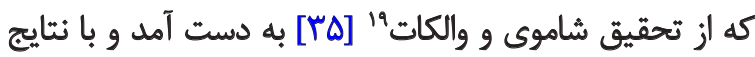

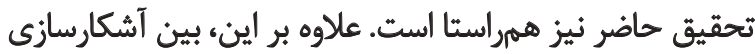

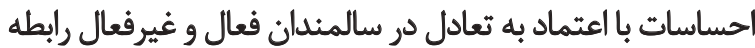

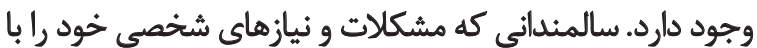

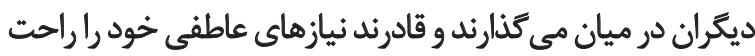

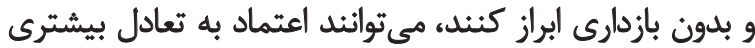

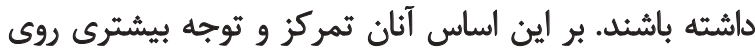

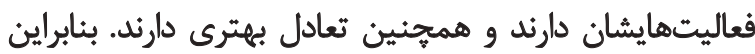

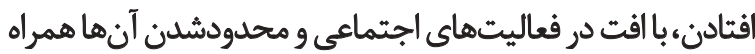

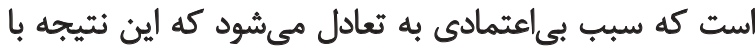

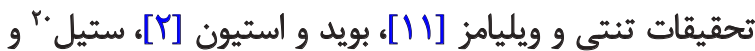

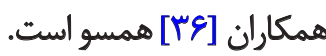

در ضمن، بين قاطعيت با اعتماد به تعادل در سالمندان

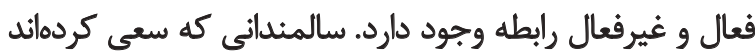

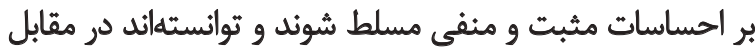

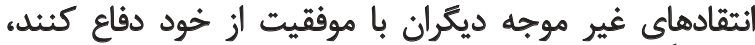

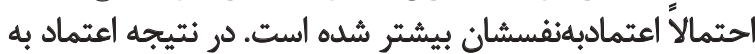

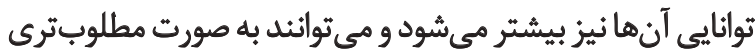

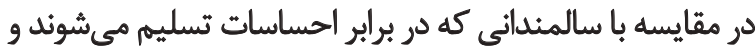

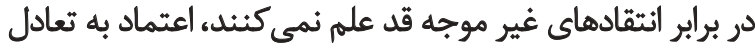

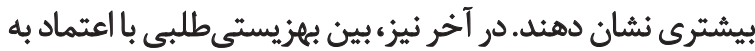
تعادل در سالمندان فعال و غير فعال رابطه وجود دارد.

كسانى كه رضايت خاطر ديگران را مدنظر قرار مى مدهند،

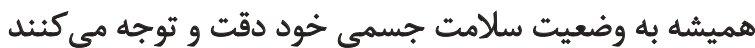

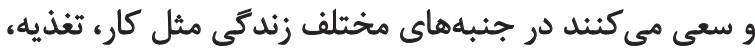

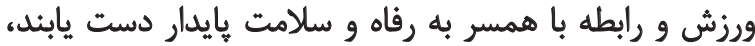

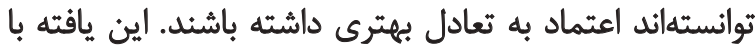

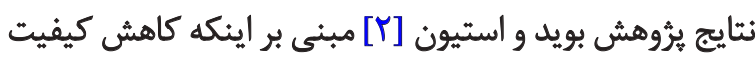

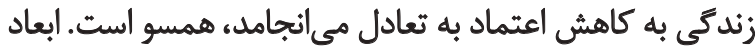

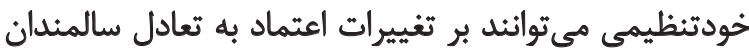

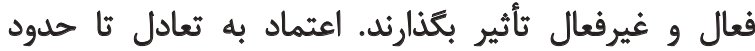

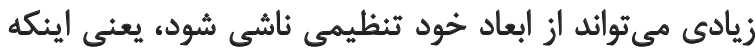

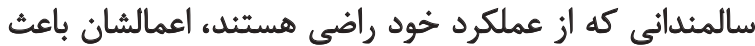

19. Shumway and Woollacott 20. Stel 


$$
\text { تشكر و قدردانى }
$$

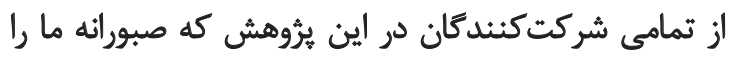

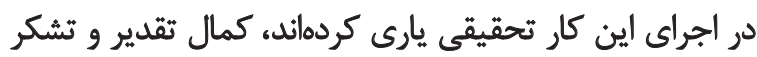

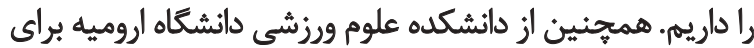

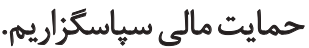

داشتند كه مي توان در يرؤوهشهاي آتى با كنترل اين متغيرها يُؤوهش هاى كسترده و جامعترى انجام داد.

\section{نتيجليَيرى نهايى}

بر اساس يافتههاى تحقيق حاضر، به ارتقاى ميزان فعاليت

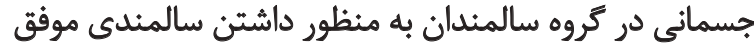

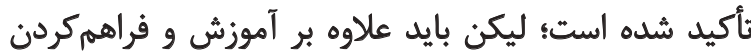

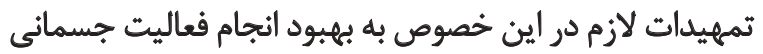

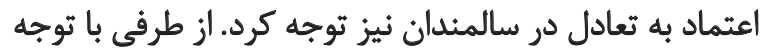

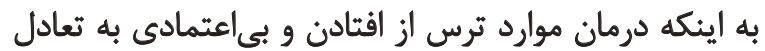

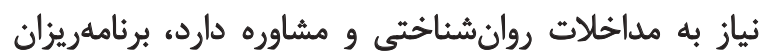

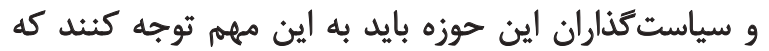

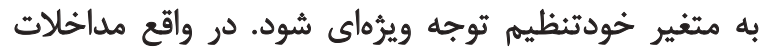

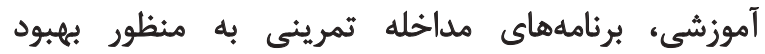

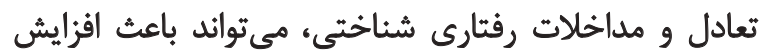

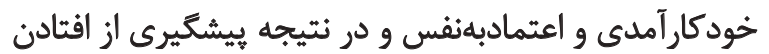

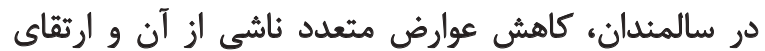

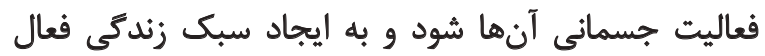

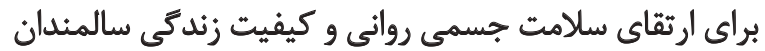
و افزايش تعداد سالمندان فعال كمك كندان

با ايجاد علاقه و انكيزه در سالمندان به انجام ورزشهاى مفرح

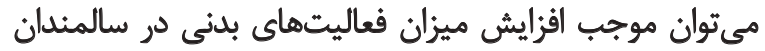

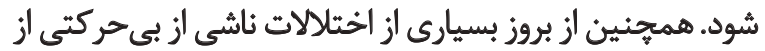

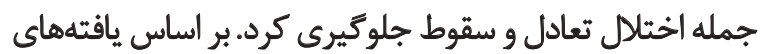

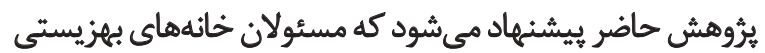

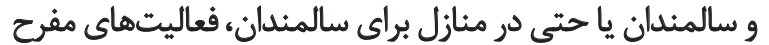

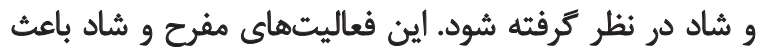

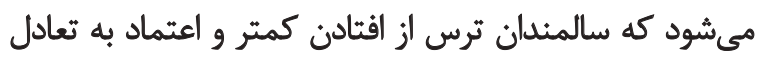

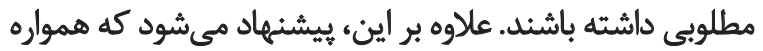

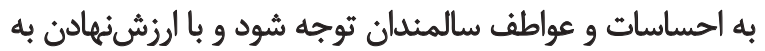

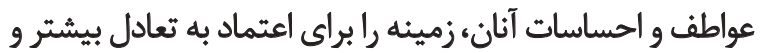
نترسيدن از افتادن تقويت كرد.

بيشنهاد ميشود كه روحيه قاطعيت و قدرت دفاع از خود

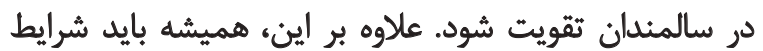

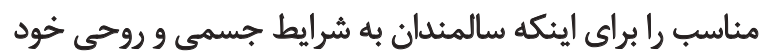

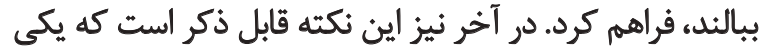

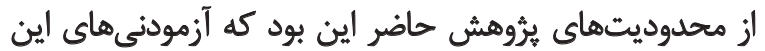

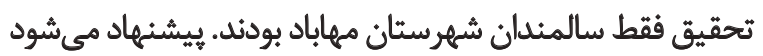

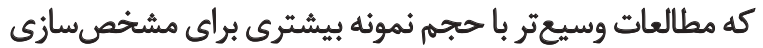

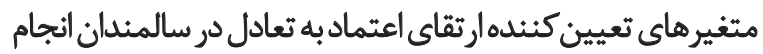

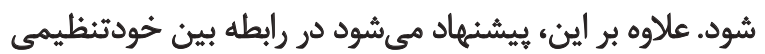

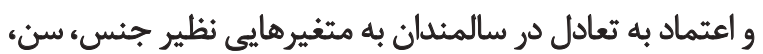
تحصيلات توجه و نتايج كستردةترى ارائه شود. 


\section{References}

[1] Zijlstra GA, Van Haastregt JC, Van Eijk JT, Van Rossum E, Stalenhoef PA, Kempen GI. Prevalence and correlates of fear of falling, and associated avoidance of activity in the general population of community-living older people. Age and Ageing. 2007; 36(3):3049. doi: 10.1093/ageing/afm021

[2] Boyd R, Stevens JA. Falls and fear of falling: Burden, beliefs and behaviours. Age and Ageing. 2009; 38(4):423-8. doi: 10.1093/ageing/afp053

[3] Close JC. Prevention of falls in older people. Disability and Rehabilitation. 2005; 27(18-19):1061-71. doi:10.1080/09638280500056469

[4] Farsi A, Ashayeri H, MohammadzadehS. [The effect of six weeks balance training program on kinematic of walking in women elderly people (Persian)]. Iranian Journal of Ageing. 2015; 9(4):27887.

[5] Seo BD, Kim BJ, Singh K. The comparison of resistance and balance exercise on balance and falls efficacy in older females. European Geriatric Medicine. 2012; 3(5):312-6. doi: 10.1016/j.eurger.2011.12.002

[6] Finlayson ML, Peterson EW, Cho CC. Risk factors for falling among people aged 45 to 90 years with multiple sclerosis. Archives of Physical Medicine and Rehabilitation. 2006; 87(9):1274-9. doi: 10.1016/j.apmr.2006.06.002

[7] Mojabi MA. [Dizziness in elderly (Persian)]. Journal of Qazvin University of Medical Sciences. 2007; 11(2):71-7.

[8] Richardson JK, Ashton-Miller JA, Lee SG, Jacobs K. Moderate peripheral neuropathy impairs weight transfer and unipedal balance in the elderly. Archives of Physical Medicine and Rehabilitation. 1996; 77(11):1152-6. doi: 10.1016/s0003-9993(96)90139-2

[9] Williams HG, McClenaghan BA, Dickerson J. Spectral characteristics of postural control in elderly individuals. Archives of Physical Medicine and Rehabilitation. 1997; 78(7):737-44. doi: 10.1016/ s0003-9993(97)90082-4

[10] Gallahue DL, Ozmun JC. Understanding motor development: Infants, children, adolescents, adults. New York: McGraw-Hill Education; 1998

[11] Tinetti ME, Williams CS. The effect of falls and fall injuries on functioning in community-dwelling older persons. The Journals of Gerontology Series A: Biological Sciences and Medical Sciences. 1998; 53(2):M112-9. doi: 10.1093/gerona/53a.2.m112

[12] Delbaere K, Crombez G, Vanderstraeten G, Willems T, Cambier D. Fear-related avoidance of activities, falls and physical frailty. A prospective community-based cohort study. Age and Ageing. 2004; 33(4):368-73. doi: /10.1093/ageing/afh106

[13] Kulmala J, Sihvonen S, Kallinen M, Alen M, Kiviranta I, Sipilä S. Balance confidence and functional balance in relation to falls in older persons with hip fracture history. Journal of Geriatric Physical Therapy. 2007; 30(3):114-20. doi: 10.1519/00139143-20071200000006

[14] Simpson JM, Worsfold C, Fisher KD, Valentine JD. The CONFbal scale: A measure of balance confidence-a key outcome of rehabilitation. Physiotherapy. 2009; 95(2):103-9. doi: 10.1016/j. physio.2008.12.004

[15] Schmeichel BJ, Zell A. Trait self_control predicts performance on behavioral tests of self_control. Journal of Personality. 2007; 75(4):743-56. doi: 10.1111/j.1467-6494.2007.00455.x
[16] Toering TT, Elferink-Gemser MT, Jordet G, Visscher C. Selfregulation and performance level of elite and non-elite youth soccer players. Journal of Sports Sciences. 2009; 27(14):1509-17. doi 10.1080/02640410903369919

[17] Barkhoff H, Heiby EM, Pagano IS. Self-regulation skills of a competitor type vs. a training champion athlete in artistic roller skating: A season long case study in elite sport competitions. The Online Journal of Sport Psychology. 2007; 9(2): 43-57.

[18] Bandura A. Self-efficacy: Toward a unifying theory of behavioral change. Psychological Review. 1977; 84(2):191-215. doi: 10.1037//0033-295x.84.2.191

[19] Buckley J, Cameron LD. Automaticity of exercise self-regulatory efficacy beliefs in adults with high and low experience in exercise self-regulation. Journal of Sport and Exercise Psychology. 2011;33(3):325-48. doi: 10.1123/jsep.33.3.325

[20] Bargh JA. Automaticity of everyday life. In: Wyer Jr RS, editor The Automaticity of Everyday Life: Advances in Social Cognition, Volume X (Advances in Social Cognition Series). London: Psychology Press; 1997.

[21] Fitzsimons GM, Bargh JA. Automatic self-regulation. In Baumeister RF, Vohs KD, editor. Handbook of Self-Regulation: Research, Theory, and Applications. New York: Guilford Press; 2004.

[22] Ibánez MI, Ruipérez MA, Moya J, Marqués MJ, Ortet G. A short version of the self-regulation inventory (SRI-S). Personality and Individual Differences. 2005; 39(6):1055-9. doi: 10.1016/j. paid.2005.02.029

[23] Khajooye D, Farokhi A, Jabiri AAM, Kazemnejad A. [Correlation between changes in balance and fall-related psychological and functional measures of men older adults after a combined training program (Persian)]. Raftar Harekati. 2015; 6(17):55-72.

[24] Mousavi A, Moghtader L .The effect of self-regulation skills on self-efficacy and happiness of high-school students .Journal of Applied Environmental and Biological Sciences. 2015; 5(12S):17982.

[25] Hatch J, Gill-Body KM, Portney LG. Determinants of balance confidence in community-dwelling elderly people. Physical Therapy. 2003; 83(12):1072-9. doi: 10.1093/ptj/83.12.1072

[26] Nemmers TM, Miller JW. Factors influencing balance in healthy community-dwelling women age 60 and older. Journal of Geriatric Physical Therapy. 2008; 31(3):93-100. doi: 10.1519/00139143200831030-00003

[27] Schepens S, Goldberg A, Wallace M. The short version of the Activities-specific Balance Confidence ( $\mathrm{ABC}$ ) scale: Its validity, reliability, and relationship to balance impairment and falls in older adults. Archives of Gerontology and Geriatrics. 2010; 51(1):9-12. doi: 10.1016/j.archger.2009.06.003

[28] Koestner R, Taylor G, Losier GF, Fichman L. Self-regulation and adaptation during and after college: A one-year prospective study. Personality and Individual Differences. 2010; 49(8):869-73 doi: 10.1016/j.paid.2010.07.019

[29] Miller DI, Taler V, Davidson PS, Messier C. Measuring the impact of exercise on cognitive aging: Methodological issues. Neurobiology of Aging. 2012; 33(3):622.e29-e43.doi: 10.1016/j.neurobiolaging.2011.02.020

[30] Al-rawahi N. Self-Regulated learning processes utilized by Omani Physical Education candidates in mastering sport skills. 
Journal of Physical Education and Sport Management. 2015; 6(4):25-31. doi: 10.5897/JPESM2015.0225

[31] Wajda DA, Roeing KL, McAuley E, Motl RW, Sosnoff JJ. The relationship between balance confidence and cognitive motor interference in individuals with multiple sclerosis. Journal of Motor Behavior. 2016; 48(1):66-71. doi: 10.1080/00222895.2015.1040110

[32] Powell LE, Myers AM. The Activities-specific Balance Confidence $(\mathrm{ABC})$ scale. The Journals of Gerontology Series A: Biological Sciences and Medical Sciences. 1995; 50(1):M28-34. doi: 10.1093/gerona/50a.1.m28

[33] Besharat MA, Bazzazian S. [Psychometri properties of the Cognitive Emotion Regulation Questionnaire in a sample of Iranian population (Persian)]. Advances in Nursing \& Midwifery. 2014; 24(84):61-70. doi: $10.22037 /$ anm.v24i84.7360

[34] Kooshyar H, Najafi, Z,Mazloom SR, Azhari A. [Comparison of the effects of exhilarating and normal physical activities on the balance and fear of falling in the elderly residing in nursing homes of Mashhad (persian)]. Evidence Based Care. 2015; 5(14):36-46.

[35] Shumway-Cook A, Woollacott MH. Motor control: Translating research into clinical practice. Philadelphia, Pennsylvania: Lippincott Williams \& Wilkins; 2007.

[36] Stel VS, Smit JH, Pluijm SM, Lips P. Consequences of falling in older men and women and risk factors for health service use and functional decline. Age and Ageing. 2004; 33(1):58-65. doi: 10.1093/ageing/afh028

[37] Liu-Ambrose T, Katarynych LA, Ashe MC, Nagamatsu LS, Hsu CL. Dual-task gait performance among community-dwelling senior women: the role of balance confidence and executive functions. The Journals of Gerontology Series A: Biological Sciences and Medical Sciences. 2009; 64A(9):975-82. doi: 10.1093/ gerona/glp063 